\title{
Metacognition and proofreading: the roles of aging, motivation, and interest
}

Article

Accepted Version

Hargis, M. B., Yue, C. L., Kerr, T., Ikeda, K., Murayama, K. and Castel, A. D. (2017) Metacognition and proofreading: the roles of aging, motivation, and interest. Aging, Neuropsychology and Cognition, 24 (2). pp. 216-226. ISSN 1744-4128 doi:

https://doi.org/10.1080/13825585.2016.1182114 Available at https://centaur.reading.ac.uk/65627/

It is advisable to refer to the publisher's version if you intend to cite from the work. See Guidance on citing.

To link to this article DOI: http://dx.doi.org/10.1080/13825585.2016.1182114

Publisher: Taylor \& Francis

All outputs in CentAUR are protected by Intellectual Property Rights law, including copyright law. Copyright and IPR is retained by the creators or other copyright holders. Terms and conditions for use of this material are defined in the End User Agreement.

\section{www.reading.ac.uk/centaur}

\section{CentAUR}

Central Archive at the University of Reading 
Reading's research outputs online 


\title{
Metacognition and Proofreading: The Roles of Aging, Motivation, and Interest
}

\author{
Mary B. Hargis, Carole L. Yue, Tyson Kerr, Kenji Ikeda, Kou Murayama, \& Alan D. Castel \\ University of California, Los Angeles
}

$\underline{\text { In Press - Aging, Neuropsychology and Cognition }}$

\begin{abstract}
Author Note
Mary B. Hargis, Carole L. Yue, Tyson Kerr, Kenji Ikeda, Kou Murayama, \& Alan D. Castel

Department of Psychology, University of California, Los Angeles.

This work was supported in part by the National Institutes of Health (National Institute on Aging), Award Number R01AG044335.

Please address all correspondence to Alan D. Castel, Department of Psychology, University of California, Los Angeles, 1285 Franz Hall Box 951563, Los Angeles, CA 90095, email: castel@ucla.edu or Mary B. Hargis, Department of Psychology, University of California, Los Angeles, 1285 Franz Hall Box 951563, Los Angeles, CA 90095, mbhargis@g.ucla.edu
\end{abstract}




\begin{abstract}
Objectives: The current study examined younger and older adults' error detection accuracy, prediction calibration, and postdiction calibration on a proofreading task, to determine if agerelated difference would be present in this type of common error detection task.
\end{abstract}

Method: Participants were given text passages, and were first asked to predict the percentage of errors they would detect in the passage. They then read the passage and circled errors (which varied in complexity and locality), and made postdictions regarding their performance, before repeating this with another passage and answering a comprehension test of both passages.

Results: There were no age-related differences in error detection accuracy, text comprehension, or metacognitive calibration, though participants in both age groups were overconfident overall in their metacognitive judgments. Both groups gave similar ratings of motivation to complete the task. The older adults rated the passages as more interesting than younger adults did, although this level of interest did not appear to influence error-detection performance.

Discussion: The age equivalence in both proofreading ability and calibration suggests that the ability to proofread text passages and the associated metacognitive monitoring used in judging one's own performance are maintained in aging. These age-related similarities persisted when younger adults completed the proofreading tasks on a computer screen, rather than with paper and pencil. The findings provide novel insights regarding the influence that cognitive aging may have on metacognitive accuracy and text processing in an everyday task.

Keywords: aging, metacognition, proofreading, motivation, comprehension 
Proofreading is a necessary skill in many occupations, and many people have high confidence in their ability to detect errors in written work. Being an inaccurate proofreader, especially if the reader is unaware of his or her lack of skill, can lead to embarrassing or costly mistakes. In the current study, younger and older adults predicted how well they would perform on a proofreading task, marked all the errors they found in passages that contained four types of grammatical and spelling errors, and then made postdiction judgments of their performance. Additionally, participants answered comprehension questions to test their memory for facts that were critical to passage comprehension and for minor details, and rated how interesting they found the passages, as well as their motivation to complete the task.

Metacognitive monitoring, or the ability to keep track of the present state of the cognitive system, is thought to be a largely preserved ability in aging (Hertzog \& Hultsch, 2000). While older adults may face deficits in various controlled processing tasks, they are often able to accurately monitor their own performance (Hertzog \& Dunlosky, 2011; Hertzog \& Hultsch, 2000). If monitoring accuracy is preserved, it may support older adults' use of strategies to compensate for their losses in other domains, such as episodic memory (Hertzog \& Dunlosky, 2011), as well as assist them in learning effective strategies through task experience (Tullis \& Benjamin, 2012) and guessing about the source of information (Kuhlmann \& Touron, 2010). Hertzog (2002) suggests that although predictions are often weakly correlated with task performance at first (if they are correlated at all), participants of all ages can learn through task experience to become better tuned to their abilities, and therefore give more accurate predictions of future performance (though the manipulation of cognitive monitoring may consume significant resources; Stine-Morrow, Shake, Miles, \& Noh, 2006). 
However, metacognition - specifically when defined as the ability to accurately monitor cognitive lapses - may not be without fault in older adults (Mecacci \& Righi, 2006; Pansky, Goldsmith, Koriat, \& Pearlman-Avnion, 2009). Even younger adults, who have not yet experienced age-related decline of cognitive processing resources, are often highly overconfident in predicting and postdicting their scores on class exams (Hacker, Bol, Horgan, \& Rackow, 2000; see also Miller \& Geraci, 2011). Overconfidence in future performance can lead to suboptimal resource allocation, either during preparation (e.g., studying for the exam), or during the task itself (i.e., taking the exam). In laboratory-based memory tasks, older adults are often less accurate at making predictions of their performance than postdictions, suggesting that they may be underconfident or overconfident before the task, but do monitor their performance during the task itself (Devolder, Brigham, \& Pressley, 1990; cf. Halamish, McGillivray, \& Castel, 2011). Prior work has not fully determined, however, if older adults can accurately predict and/or postdict their performance on a task that they may encounter in real life (as many younger adults struggled to do in Hacker, Bol, Horgan and Rackow, 2000).

Completely avoiding writing errors may be impossible regardless of age, but being able to take note of and correct mistakes requires skills that may be preserved across the lifespan. Linguistic knowledge is relatively preserved in aging (Koss et al., 1991), and older adults often outperform their younger counterparts on vocabulary tests, especially when those tests are in multiple choice format (though age differences tend to disappear when education level is accounted for; Verhaeghen, 2003). Older adults perform well on proofreading and text comprehension tasks when those skills are examined individually, providing evidence for their preservation through the lifespan. 
Madraso (1993) suggests that intact working memory is critical and necessary to proofread a text passage correctly, as some grammatical rules are applied across sentences, and the words that must agree (e.g., a subject and a verb) may be separated by many chunks of information. Older adults can face significant deficits in working memory (Salthouse, 1990), suggesting that their identification of errors that can only be detected when considering the sentence as a whole may be less accurate than that of younger adults. In the current task, participants are required to comprehend the content of a sentence (which is necessary to identifying global errors, such as subject-verb disagreement) while also paying attention to lower-level spelling errors that may not be connected to more global content. Previous work on age-related cognitive control deficits suggests that older adults' ability to accurately detect global errors and their performance on the final surprise comprehension task may suffer as compared to younger adults, as proofreading and comprehension require distinct cognitive processes, which older adults may not use simultaneously and spontaneously (i.e., without prior knowledge of a comprehension test; Connelly, Hasher, \& Zacks, 1991; Verhaeghen Steitz, Sliwinski, \& Cerella, 2003).

Alternatively, the superior linguistic knowledge and vocabulary of older adult readers could be put to use in detection of various types of errors (Daneman, Hannon, \& Burton, 2006). In prior work (Daneman, Hannon, \& Burton, 2006), younger and older adults were both equally susceptible to shallow processing of sentences in which they were asked to detect anomalies (e.g., the phrase "surviving dead"), suggesting that age-related differences may not be present in the detection of deeper-level errors. Additionally, the ability to comprehend text may be relatively preserved in aging (De Beni, Palladino, Borella, \& Presti, 2003; cf. Dunlosky, Baker, Rawson, \& Hertzog, 2006), though prior work has also suggested that older adults may have 
processing difficulties due to deficits in working memory (Stine, 1990; see also the levels-ofprocessing hypothesis, Craik \& Tulving, 1975). It is unclear whether comprehension for text will suffer if attention is focused elsewhere during the primary task - for example, on finding possible grammatical or spelling errors in the passage, as in the current study.

The goal of the current study was to examine how proofreading accuracy and metacognitive monitoring of performance may or may not change across the lifespan, and whether interest, comprehension, and motivation are notable influences on performance on a task that requires older adults to use skills that may be preserved in aging. To examine these factors, participants made predictions and postdictions of their performance (as measured by percent of errors detected) on each of two passages they were asked to proofread. Participants also rated how interesting they found each passage and their overall motivation to perform well on the task, and answered surprise comprehension questions to gauge their general and specific understanding of the information in each passage. The current study also sought to investigate the hypothesis that older participants' interest and motivation may positively affect their scores on the task enough to overcome their working memory deficits, as interesting passages are comprehended with more accuracy (Hidi, 1990) and an individual's high motivation leads to better task performance (Locke, 1968; cf. McGillivray, Murayama, \& Castel, in press); though, alternatively, it may be that too much interest in a passage may cause proofreading accuracy to suffer (as "seductive details" may become distractions; Harp \& Mayer, 1998).

\section{Method}

\section{Participants}

Thirty-one older adults $\left(M_{\mathrm{age}}=78.0\right)$ recruited from the community participated for $\$ 10$ per hour. Thirty-one younger adults $\left(M_{\mathrm{age}}=20.2\right)$, were undergraduates at the University of 
California, Los Angeles, and participated for course credit. Older adults had completed an average of 16.1 years of education $(S D=2.26)$, and younger adults had completed an average of 14.7 years of education $(S D=1.14)$.

\section{Materials and Procedure}

Before participants began the proofreading experiment, they were told that they would be proofreading a text with "spelling errors, verb tense errors, and other typographical errors" and were asked to make a prediction of what percentage (out of 100\%) of those errors they would be able to correctly detect. Participants were then given a paper copy of two out of the four passages on the following topics: the habitat and lifestyle of elephants, the Battle of Chaldiran, the Albert Bridge in England, and the history of criminal law. Three passages were taken from Wikipedia entries, and the fourth (the history of criminal law) was from a Graduate Record Examination (GRE) Comprehension section practice website. Passage length ranged from 205-254 words, with a mean length of 235.75 words. The Gunning Fog index of the passages (a measure of text readability which estimates the number of years of education needed to comprehend a text) ranged from 14.3 to $15.8(M=15.2, S D=.67)$, which was judged as an education-appropriate level for the participants in this study. Each passage contained four errors of each of the following types, as adapted from Brunyé, Mahoney, Rapp, Ditman, and Taylor (2011): simple local errors (misspellings of 1-2 syllable words), complex local errors (misspellings of 3-4 syllable words), simple global errors (homophones), and complex global errors (subject-verb agreement or verb tense). (See Appendix for excerpts and sample errors from each passage.) Participants received one passage at a time (chosen randomly) to read for three minutes, and the presentation order and combinations of articles were counterbalanced. Participants were instructed to circle any words that they believed were incorrect or inappropriate in the context of 
the passage and to avoid circling correct words or phrases. The errors occurred only within words, not in punctuation or across sentences. Participants were not asked to write in the correct word or words, only to indicate the errors. Once participants completed their proofreading of the first passage, they immediately made a postdiction judgment on the percentage of errors they had detected (out of 100\%) and then rated how interesting they found the passage, on a scale from 110. This procedure (prediction, proofreading, postdiction, interest rating) was repeated for a second passage. After completing both passages, participants answered a series of eight multiplechoice comprehension questions (four questions on each passage) to test their memory for facts or ideas that were critical for the comprehension of the passage (e.g., "Why was the Battle of Chaldiran of major historical importance?") and for minor details (e.g., "Approximately how many executions per year were there in $16^{\text {th }}$ century England?").

\section{Results}

\section{Error Detection}

There were no significant differences between younger and older adults in proofreading accuracy. A 2 (passage 1 , passage 2$) \times 2$ (younger adults, older adults $) \times 4$ (error type) ANOVA showed no effect of age on error detection accuracy, $F<1, \eta_{\mathrm{p}}^{2}=.01$. There was no significant three-way interaction; $F<1, p=.37, \eta_{\mathrm{p}}^{2}=.02$. The two-way interaction between error type and age was also insignificant, $F(3,180)<2.36, p=.07, \eta_{\mathrm{p}}^{2}=.04$, as was the two-way interaction between passage and age group, $F<1, \eta_{\mathrm{p}}^{2}=.01$, and the two-way interaction between error type and passage, $F(3,180)=1.05, p=.29, \eta_{\mathrm{p}}^{2}=.02$. The main effect of passage number was not statistically significant, $F(1,60)=2.31, p=.13, \eta_{\mathrm{p}}^{2}=.04$. There was a significant main effect of error type, $F(3,180)=30.64, p<.001, \eta_{p}^{2}=.34$ 
Pairwise comparisons using Bonferroni corrections indicated that, overall, participants correctly circled more simple local errors (misspelling of 1-2 syllable words) than complex local errors (misspellings of 3-4 syllable words, $p<.001$ ), simple global errors (homophones, $p=$ .001 ), or complex global errors (subject-verb agreements or verb tense, $p<.001$ ). Additionally, participants circled more simple global errors than complex local errors $(p=.002)$ and complex global errors $(p<.001)$. (For mean errors correctly circled, see Table 1.)

\section{Prediction, Postdiction, and Calibration}

To analyze whether participants were significantly overconfident in their prediction and/or postdiction judgments as compared to their actual performance on the proofreading task, a 2(passage 1, passage 2) X 3(prediction, actual performance, postdiction) X 2 (younger adults, older adults) ANOVA was conducted. This test revealed no significant three-way or two-way interactions, but did reveal significant differences among prediction, postdiction, and actual scores $F(2,120)=15.54, p<.001, \eta_{\mathrm{p}}^{2}=.21$. There was no significant main effect of passage, $F(1,60)=3.35, p>.07, \eta_{\mathrm{p}}^{2}=.05$, suggesting that participants' performance did not significantly change across passages. Follow-up comparisons conducted across both age groups indicated significant differences between prediction and actual score (means of 64.60 and 53.43, respectively) and postdiction and actual score (means of 65.56 and 53.43, respectively) for both passages (all $p$ s $\leq .001)$.

Calibration, or the accuracy with which participants made their predictions and postdictions, was calculated by subtracting participants' actual scores from their estimated scores on the proofreading task (as in Hacker et al., 2000). A 2(passage 1, passage 2) $\times 2$ (prediction calibration, postdiction calibration) $\times 2$ (younger adults, older adults) ANOVA revealed no effect of age on calibration, $F<1, \eta_{\mathrm{p}}^{2}=.004$, such that older adults and younger adults were 
equivalently accurate in their metacognitive judgments on this task (younger adults' mean calibration was 20.50 and older adults' mean calibration was 22.10 , with 0 being perfectly calibrated). There was no significant three-way interaction, $\mathrm{F}(1,60)=1.96, \mathrm{p}=.17, \eta_{\mathrm{p}}^{2}=.03$; or two-way interactions between prediction/postdiction and age group, $\mathrm{F}<1, \mathrm{p}=.87, \eta_{\mathrm{p}}^{2}<.001$, prediction/postdiction and passage, $F(1,60)=1.96, p=.17, \eta_{\mathrm{p}}^{2}=.03$, or age and passage, $F<$ $1, p=.638, \eta_{\mathrm{p}}^{2}<.01$. There were also no main effects of passage or prediction/postdiction, both $F \mathrm{~s}<1, p \mathrm{~s}>.33 ., \eta_{\mathrm{p}}^{2}<.02$

\section{Interest, Motivation, and Comprehension}

There was no significant age difference in participants' self-reported motivation to do well on the proofreading task; younger adults rated their motivation as 7.55 out of 10 on average $(S D=1.65)$, while older adults rated theirs as 8.19 out of $10(S D=1.78), t(60)=-1.418, p=.144$. Motivation was correlated with error detection performance on both passages for older adults, $r$ $=.532, p=.002$ for passage 1 and $r=.531, p=.002$ for passage 2. Younger adults' motivation to perform well on the task, however, was only correlated with performance on passage $1, r=.31, \mathrm{p}$ $=.04$, not with performance on passage $2, r=.32, p=.08$. There was no significant correlation between younger adults' motivation and calibration (all $p s>.25$ ) or between older adults' motivation and calibration (all $p \mathrm{~s}>.50)$.

Older adults did rate the passages as more interesting than younger adults, $F(1,60)=$ $5.418, p=.023$; younger adults rated their interest as $5.22(S D=2.04)$ on average, while older adults rated theirs as $6.44(S D=2.41)$. Interest was not correlated with performance or calibration in either age group at the $p=.05$ level; for older adults, there was a correlation of .09 between interest and performance and -.07 between interest and calibration, for younger adults, 
there was a correlation of .05 between interest and performance and -.05 between interest and calibration.

To analyze comprehension accuracy, a 2(minor questions, important questions) $\times$ 2 (young, old $) \times 2$ (passage 1 , passage 2 ) ANOVA was conducted, and found no significant threeway interaction, $F(1,60)=1.70, p=.20, \eta_{\mathrm{p}}^{2}=.03$; no significant two-way interactions between question type and age group, $F(1,60)=2.32, p=.133, \eta_{\mathrm{p}}^{2}=.04$; question type and passage, $F(1,60)=1.70, p=.20, \eta_{\mathrm{p}}^{2}=.03$; or passage and age group, $F<1, \eta_{\mathrm{p}}^{2}<.001$. There were no main effects of passage or age, both $F_{\mathrm{s}}<1, \eta_{\mathrm{p}}^{2}<.02$. There was a main effect of question type, $F(1,60)=9.83, p=.003, \eta_{\mathrm{p}}^{2}=.14$, such that questions about minor details were answered with more accuracy than questions that were important for the overall comprehension of the passage $(M=1.36$ and $M=1.08$, respectively).

\section{Discussion}

The age equivalence in both proofreading ability (as measured by error detection accuracy) and calibration (the accuracy of prediction and postdiction judgments of performance on the proofreading tasks) suggests that the ability to proofread text passages and the metacognitive monitoring used in judging one's own performance are maintained in aging. Participants were overconfident in their predictions and postdictions overall, though effect sizes were small. There are no significant differences in the number of errors older adults detected in each passage as compared to younger adults. The lack of age differences in proofreading accuracy suggests that metacognitive inaccuracy is not due to age differences in performance. In the current study, the primary measure on which younger and older adults differed was the interest rating each age group gave to the text passages (older adults rated the passages as significantly more interesting than younger adults, giving an average rating of 6.44 as opposed to 
younger adults' average rating of 5.23 out of 10). Since older adults' performance was not negatively associated with their higher interest in the passages, it seems that the passages were not so interesting as to distract participants from the tasks at hand (i.e., proofreading the passage and understanding its content).

Similar to Hacker et al. (2000), participants were overconfident in their prediction and postdiction judgments for both passages. It seems that participants did not update their metacognitive judgments within one passage (i.e., after completing the proofreading task) or between passages (i.e., having task experience with a prediction-proofreading-postdiction session already completed), which is inconsistent with Dunlosky and Hertzog (2000), in which participants became more accurate in their metacognitve judgments with task experience. However, Dunlosky and Hertzog (2000) did find that difference scores contrasting performance with predictions and postdictions were worse for the second list than the first list, such that people became more underconfident in their performance when using interactive imagery to study paired-associates (see also Hertzog et al., 2009; Price, Hertzog, \& Dunlosky, 2008). In the present study, it may be necessary to have direct feedback or several trials (more than just two) in which participants become more aware of their performance, and what factors influence the strategies that are used during proofreading and when making metacognitive judgments. It is also important to note that metacognitive judgments were not given for each type of error (simple and complex, global and local), though this would be an appropriate avenue for future research; rather, pre- and postdictions were used, assessing more global metacognition.

Prior work presented multiple alternatives for the outcome of the current study: namely, that the working memory load of detecting global errors would cause older adults' proofreading performance to suffer compared to younger adults, or that older adults' preserved comprehension 
and verbal abilities would promote accurate performance on both tasks even when using working memory. In the current study, there were no age differences in proofreading or comprehension accuracy between younger and older adults, supporting the idea of preserved text comprehension abilities in aging put forth in De Beni et al. (2003), even though some questions were detailoriented (as opposed to gist-based) which can present problems for older adults (Koutstaal \& Schacter, 1997; Brainerd \& Reyna, 2001). There is some evidence that shallow processing of proofreading and comprehension is dominant in both age groups, as in Daneman, Hannon, and Burton (2006): the errors that require shallower processing in proofreading (simple local and simple global errors) were detected with more accuracy than the deeper grammatical errors, and the comprehension questions that required shallower processing by testing memory for minor details of the passage were answered more accurately than the information that was more important to overall understanding of the passage. Additionally, interest in the passage may benefit older adults in the comprehension test, but does not distract attention enough to decrease proofreading accuracy. Younger adults' motivation to perform well was positively correlated with their proofreading performance on passage 1 but not passage 2, while older adults' motivation was positively correlated with their proofreading performance on both passages. This, along with the lack of correlation between motivation and calibration for either age group, suggests that high task motivation is only related to performance on the primary task, and does not have any further connection to metacognitive measures such as predictions and postdictions.

It is worth noting that participants in this study edited paper copies of text passages, though using electronic word processors to write and edit documents may now be more common for both age groups, and perhaps especially so for younger adults. In order to ensure the lack of age-related differences in detecting errors in the present study was not due to any potential 
underestimates of performance due to use of paper-based testing for younger adults, we conducted a follow-up study, as it may be the case that younger adults would perform better in a "more typical” computer-screen viewing environment, In our follow-up study, , we recruited younger adults $\left(n=31, M_{\text {age }}=20.74,11\right.$ males $)$ who completed the same task with the same stimuli presented on a computer screen (instead of on paper, as in the study reported above). We found that younger adults who completed the task on paper were, in fact, more accurate in detecting errors than those who completed the task on a computer, $F(1,60)=4.91, p=.03, \eta_{\mathrm{p}}^{2}=$ .08 , though there were no differences between those groups in metacognitive calibration, $F(1,60)$ $=.21, p=.65, \eta_{\mathrm{p}}^{2}<.01$. This reinforces findings that younger people are more accurate in performing proofreading tasks on paper rather than on a screen, and provides additional support for the lack of age differences in the current study as being truly representative of a broader population. Thus, the findings from this follow-up study are consistent with previous work that has shown that younger adults are more accurate in proofreading an unedited passage if that text is printed out and given to them in hard copy, as opposed to viewed and edited on the computer (Wharton-Michael, 2008). Thus, it appears to be the case that there are benefits with doing things the "old fashioned way" - that is, editing written communication on paper instead of on a screen - aids in error detection.

The findings from the present study fit with other research that shows some age-related preservation of function with verbal tasks and metacognitive monitoring (see Castel, Middlebrooks, \& McGillivray2015; Hertzog \& Dunlosky, 2011). It may be that older adults have sufficient experience with proofreading and detecting errors in text, and this type of skill is maintained with age, even for text that is interesting as could potentially distract one from the task of finding spelling errors. We do note that future research is needed in this domain, as some 
of the present conclusions rest on the null effects of age, likely with a larger sample given the small effect size reported in the present study, and with a greater variety of proofreading tasks and baseline measures such as fluid and crystallized intelligence, working memory, vocabulary, level of reading experience, and print exposure (Stanovich \& West, 1989). While this study may represent a measurable but small step forward in the understanding of the underlying phenomena, it utilizes a novel paradigm with both younger and older adults, and the similarities between the age groups in proofreading accuracy and metacognitive calibration passages bode well for older adults. Those who are actively engaged in writing and editing their own communication may have preserved abilities as they age, though overconfidence could lead to errors going unnoticed. Reviewing a paper or an email is a metacognitively and attentionally demanding task, but cognitive control and working memory deficits do not appear to supersede older adults' preserved linguistic abilities.

Word count: 3877 


\section{References}

Brainerd, C. J., \& Reyna, V. F. (2001). Fuzzy-trace theory: Dual-processes in reasoning, memory, and cognitive neuroscience. Advances in Child Development and Behavior, 28, 49-100.

Brunyé, T. T., Mahoney, C. R., Rapp, D. N., Ditman, T., \& Taylor, H. A. (2012). Caffeine enhances real-world language processing: Evidence from a proofreading task. Journal of Experimental Psychology: Applied, 18, 95-108.

Castel, A. D., Middlebrooks, C. D., \& McGillivray, S. (2015). Monitoring memory in old age: Impaired, spared, and aware. In J. Dunlosky \& S. Tauber (Eds.), The Oxford Handbook of Metamemory. Oxford University Press.

Connelly, S. L., Hasher, L., \& Zacks, R. T. (1991). Age and reading: the impact of distraction. Psychology and Aging, 6, 533-541.

Craik, F. I., \& Tulving, E. (1975). Depth of processing and the retention of words in episodic memory. Journal of Experimental Psychology: General, 104, 268-294.

Daneman, M., Hannon, B., \& Burton, C. (2006). Are there age-related differences in shallow semantic processing of text? Evidence from eye movements. Discourse Processes, 42, 177203.

De Beni, R., Palladino, P., Borella, E., \& Presti, S. L. (2003). Reading comprehension and aging: does an age-related difference necessarily mean impairment? Aging Clinical and Experimental Research, 15, 67-76.

Devolder, P. A., Brigham, M. C., \& Pressley, M. (1990). Memory performance awareness in younger and older adults. Psychology and Aging, 5, 291-303. 
Dunlosky, J., Baker, J., Rawson, K. A., \& Hertzog, C. (2006). Does aging influence people's metacomprehension? Effects of processing ease on judgments of text learning. Psychology and Aging, 21, 390-400.

Dunlosky, J., \& Hertzog, C. (2000). Updating knowledge about encoding strategies: A componential analysis of learning about strategy effectiveness from task experience. Psychology and Aging, 15, 462-474.

Hacker, D. J., Bol, L., Horgan, D. D., \& Rakow, E. A. (2000). Test prediction and performance in a classroom context. Journal of Educational Psychology, 92, 160-170.

Halamish, V., McGillivray, S., \& Castel, A. D. (2011). Monitoring one's own forgetting in younger and older adults. Psychology and Aging, 26, 631-635.

Harp, S. F., \& Mayer, R. E. (1998). How seductive details do their damage: A theory of cognitive interest in science learning. Journal of Educational Psychology, 90, 414-434.

Hertzog, C. (2002). Metacognition in older adults: implications for application. In Perfect, T.J. \& Schwartz, B.L. (2002). Applied Metacognition. Cambridge University Press, 169-196.

Hertzog, C., \& Dunlosky, J. (2011). Metacognition in later adulthood: spared monitoring can benefit older adults' self-regulation. Current Directions in Psychological Science, 20, 167173.

Hertzog, C., \& Hultsch, D. F. (2000). Metacognition in adulthood and old age. In Craik, F. I., \& Salthouse, T. A. (Eds.). The Handbook of Aging and Cognition. Psychology Press, 417-466. Hertzog, C., Price, J., Burpee, A., Frentzel, B. J., Feldstein, S., \& Dunlosky, J. (2009). Why do people show minimal knowledge updating with task experience: Inferential deficit or experimental artifact? Quarterly Journal of Experimental Psychology, 62, 155-173. 
Hidi, S. (1990). Interest and its contribution as a mental resource for learning. Review of Educational Research, 60, 549-571.

Koss, E., Haxby, J. V., DeCarli, C., Schapiro, M. B., Friedland, R. P., \& Rapoport, S. I. (1991). Patterns of performance preservation and loss in healthy aging. Developmental Neuropsychology, 7, 99-113.

Locke, E. A. (1968). Toward a theory of task motivation and incentives. Organizational Behavior and Human Performance, 3, 157-189.

Koutstaal, W., \& Schacter, D. L. (1997). Gist-based false recognition of pictures in older and younger adults. Journal of Memory and Language, 37, 555-583.

Kuhlmann, B. G., \& Touron, D. R. (2011). Older adults' use of metacognitive knowledge in source monitoring: Spared monitoring but impaired control. Psychology and Aging, 26, 143149.

McGillivray, S., Murayama, K., \& Castel, A.D. (in press). Thirst for knowledge: The effects of curiosity and interest on memory in younger and older adults. Psychology and Aging.

Mecacci, L., \& Righi, S. (2006). Cognitive failures, metacognitive beliefs and aging. Personality and Individual Differences, 40, 1453-1459.

Miller, T. M., \& Geraci, L. (2011). Unskilled but aware: reinterpreting overconfidence in lowperforming students. Journal of Experimental Psychology: Learning, Memory, and Cognition, 37, 502-506.

Pansky, A., Goldsmith, M., Koriat, A., \& Pearlman-Avnion, S. (2009). Memory accuracy in old age: Cognitive, metacognitive, and neurocognitive determinants. European Journal of Cognitive Psychology, 21, 303-329. 
Price, J., Hertzog, C., \& Dunlosky, J. (2008). Age-related differences in strategy knowledge updating: Blocked testing produces greater improvements in metacognitive accuracy for younger than older adults. Aging, Neuropsychology, and Cognition, 15, 601-626.

Salthouse, T. A. (1990). Working memory as a processing resource in cognitive aging. Developmental Review, 10, 101-124.

Stanovich, K. E., \& West, R. F. (1989). Exposure to print and orthographic processing. Reading Research Quarterly, 402-433.

Stine, E. L. (1990). On-line processing of written text by younger and older adults. Psychology and Aging, 5, 68-78.

Stine-Morrow, E. A., Shake, M. C., Miles, J. R., \& Noh, S. R. (2006). Adult age differences in the effects of goals on self-regulated sentence processing. Psychology and Aging, 21, 790803.

Tullis, J. G., \& Benjamin, A. S. (2012). The effectiveness of updating metacognitive knowledge in the elderly: Evidence from metamnemonic judgments of word frequency. Psychology and Aging, 27, 683-690.

Verhaeghen, P. (2003). Aging and vocabulary score: A meta-analysis. Psychology and Aging, 18, 332-339.

Verhaeghen, P., Steitz, D. W., Sliwinski, M. J., \& Cerella, J. (2003). Aging and dual-task performance: a meta-analysis. Psychology and Aging, 18, 443-460.

Wharton-Michael, P. (2008). Print vs. computer screen: Effects of medium on proofreading accuracy. Journalism \& Mass Communication Educator, 63, 28-41. 


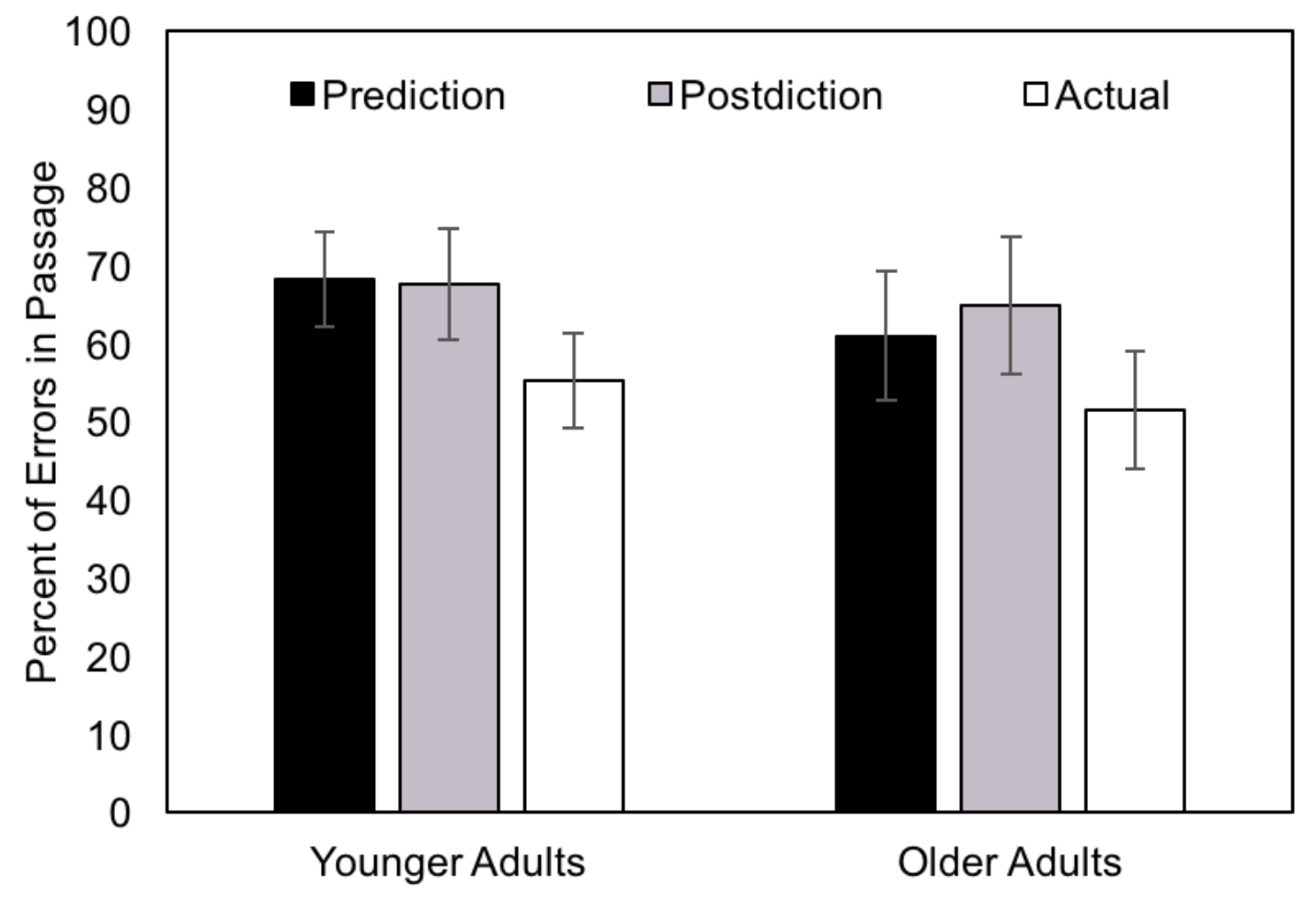

Figure 1. Younger and older adults' average prediction ratings, postdiction ratings, and actual performance on a proofreading task, averaged across two passages. Error bars represent $95 \%$ confidence intervals. 


\begin{tabular}{|c|c|c|c|c|}
\hline \multirow[b]{3}{*}{ Error Type } & \multicolumn{2}{|c|}{$\underline{\text { Younger Adults }}$} & \multicolumn{2}{|l|}{$\underline{\text { Older Adults }}$} \\
\hline & Passage 1 & Passage 2 & Passage 1 & Passage 2 \\
\hline & $M(S D)$ & $M(S D)$ & $M(S D)$ & $M(S D)$ \\
\hline Simple Local & $2.90(1.16)$ & $2.90(1.16)$ & $2.68(1.11)$ & $2.87(.96)$ \\
\hline Complex Local & $2.29(1.64)$ & $1.51(1.21)$ & $1.90(1.14)$ & $2.03(1.38)$ \\
\hline Simple Global & $2.29(1.27)$ & $2.64(1.11)$ & $2.13(1.31)$ & $2.39(1.28)$ \\
\hline Complex Global & $1.64(1.28)$ & $1.90(1.27)$ & $1.23(1.48)$ & $1.26(1.18)$ \\
\hline Total Errors & $8.74(3.17)$ & $8.97(2.66)$ & $7.94(3.67)$ & $8.55(3.52)$ \\
\hline
\end{tabular}

Table 1. The means and standard deviations of number of Simple Local, Complex Local, Simple Global, Complex Global, and Total errors correctly circled by each age group. 


\begin{abstract}
Appendix
Excerpts from each text passage used in the experiment are included below. Information in brackets indicates the type of error and were not included in the text presented to participants.
\end{abstract}

Crime: "As primitive societies developed, the government, represented by the cheif [simple local] or the king, gradually began taking over the protection of persens [simple local] and property and the punishment of offenders in the name of public piece [simple global] and order. The basic concept behind the intervention of government, however, continued to be that of retribution: a balancing of the scales of justice. The scales tended to be balanced on the side of the superior power of the state. The deth penalty was the most common response to common crime."

Bridge: "The Albert Bridge is a rode [simple global] bridge over the River Thames in West London, connecting Chelsea on the north bank to Battersea on the south bank. Designed and bilt [simple local] by Rowland Mason Ordish in 1873 as an Ordish-Lefeuvre system-modifeid [complex local], cabel-stayed [simple local] bridge, it proven [complex global] to be structurally unsound, and so between 1884 and 1887 Sir Joseph Bazalgette incorporates some of the design elements of a suspension bridge."

Elephants: "Elephants are herbavorous [complex local] and can be found in different habitats including savanahs [complex local], forests, deserts and marshes. They prefer to stay near water. They are considered to be keystone speceis [simple local] due to there [simple global] impact on 
their enviroments [complex local]. Other animals tend to keep their distance, and predators such as lions, tigers, hyenas and wild dogs usually target only the young elephants (or "calves"). Females ("cows") tends [complex global] to live in family groups, which can concist [simple local] of one female with her calves or several related females with offspring."

Battle: "The Battle of Chaldiran or Chaldoran occurred on August 23, 1514 and ended with a victory for the Ottoman Empire over the Safavid Empire. As a result, the Ottomans have gained [complex global] immediate control over eastern Anatolia and northern Iraq. The battle, however, was just the begining [complex local] of 41 years of destructive war between the too [simple global] empires that only ended in 1555 with the Traety [simple local] of Amasya. While the Ottomans often had the upper hand, the Persians for the most part hold [complex global] their ground." 